\title{
A decision-support model for operational planning of surface coal mining considering equipment failure
}

\author{
Hao Yu, Wei Deng Solvang and Xu Sun \\ Department of Industrial Technology \\ Faculty of Engineering Science and Technology \\ UiT - The Arctic University of Norway \\ Narvik, Norway \\ E-Mail: hao.yu@uit.no,wei.d.solvang@uit.no
}

\begin{abstract}
This paper presents a decision-support model aiming, primarily, at maximizing the productivity of surface coal mining activities through operational planning and management. The model is formulated based upon mixed integer programming, and it determines the optimal production plan of the shovels and allocation plan of the haul trucks in order to minimize operational cost, fuel consumption as well as greenhouse gas (GHG) emission of surface coal mining. The model is further extended to multiscenario decision aided system for taking into account of the uncertainties associated with equipment failure. Illustration and numerical calculation are given to shown the application of the proposed model. The model formulates a simplified decision making in surface coal mining operations, suggestions for further development is also given latter in this paper.
\end{abstract}

Keywords-surface coal mining; truck allocation problem; uncertainty; equipment failure; optimization; mathematical programming; operational planning and management; decision making

\section{INTRODUCTION}

Coal mining is one of the primary industries, which provides the most important resources for energy production and space heating. Coal mining includes two types of operations: Surface mining (or open-pit mining) and underground mining (or in-pit mining), and the choice of the types of operation is largely determined by the depth and geological structure of coal deposit. Today, surface mining is more common in coal mining industry due to its lower cost comparing to the underground operations.

Shovels and haul trucks are fundamental components for extracting, loading and transporting the mineral resources in coal mining system. And it is tremendously acknowledged that the daily operation of shovels and haul trucks not only takes the most significant share in the operational cost of coal mining activities [1, 2], but also becomes the largest contributor to greenhouse gas (GHG) emission [3-5]. Due to this reason, the optimization of shovel and haul truck operation is of importance to improve the profitability in coal mining industry. In the past few years, the cost saving of shovel and haul truck operation is mainly achieved through applying larger equipment or increasing the number of equipment, which aims at taking advantage of economy of scale [6]. However, this method has been proved to be inefficient and may also lead to the loss of productivity due to the likelihood of increased queue time [7]. Therefore, in recent years, coal mining companies spend more effects in the operational planning and management of shovels and haul trucks in order to improve the resource utilization and reduce the operational cost, fuel consumption as well as GHG emission from surface mining activities.

Some of the previous studies related to the operational problems in mining industry are reviewed. Norgate and Haque [3] investigated the energy consumption and GHG emission of mining activities with the help of life cycle assessment. The result reveals that the operation of shovels and haul trucks contribute the most significant share of GHG emission in surface mining. Siami-Irdemoosa and Dindarloo [4] applied artificial neural network (ANN) to predict and estimate the fuel consumptions of haul trucks in mining operations. Sahoo et al. [5] developed a generic model for assessing the fuel and energy consumption of hauling trucks in mining activities.

Application of emerging technologies in mining operations has been focused in previous studies. Mishra et al. [8] discussed the implementation of Radio Frequency Identification (RFID) technology in mining operations. Sun et al. [9] proposed a conceptual framework of an integrated information system for improving the driving safety under poor visibility conditions of mining operations. Safety is one of the most significant challenges in mining industry, and the proposed information system incorporates GIS, real-time positioning technologies, wireless communication as well as advanced algorithm in order to improve the visibility and decision-making under severe visibility condition in mining activities.

Optimization of shovel-truck problem is another focus of previous studies. Souza et al. [10] developed a hybrid heuristic algorithm to optimize the allocation and management of shovels and haul trucks in surface mining operations. Rodrigo et al. [11] formulated an optimization model for shovel-truck operation in surface mining system. The model aims at maximizing the productivity of mining operations while it is restricted by equipment availability and dynamic constraints. Ta et al. [12] proposed a linear programming model for shovel-truck problem,

“Smart Mining Technology” project (No. 2014-0083). 
in which the idle probabilities of equipment is taken into consideration.

In this paper, the shovel-truck planning problem in a surface coal mining system is first formulated and validated. The model will then be extended to a multi-scenario decision aided model which takes into account of the uncertainties related to equipment failure.

The reminder of the paper is organized as follows. Section II introduces the shovel production and truck allocation problem, and the mathematical model is also formulated in this section. Section III provides illustration of the proposed mathematical model. Section IV extends the model to a multi-scenario decision making model in order to take into consideration of the influence of equipment failure, and the comparative calculation is also given in this section. Section $\mathrm{V}$ summarizes the limitation of this study and gives suggestions of future development.

\section{THE MODEL}

\section{A. Problem description}

Shovel-truck operation is the most expensive activity in surface mining and therefore needs to be optimized in order to improve the profitability and reduce the cost and GHG emission. Besides, in coal mining operations, there are many uncertainties existed, i.e., shovel or haul truck breakdowns, etc., which may heavily influence the workflow. In order to resolve those problems, managers in coal mining companies usually allocate more trucks than necessary, which increases the operating cost and creates waste such as inefficient use of equipment. This section discusses an optimal shovel production and truck allocation problem on a daily basis.

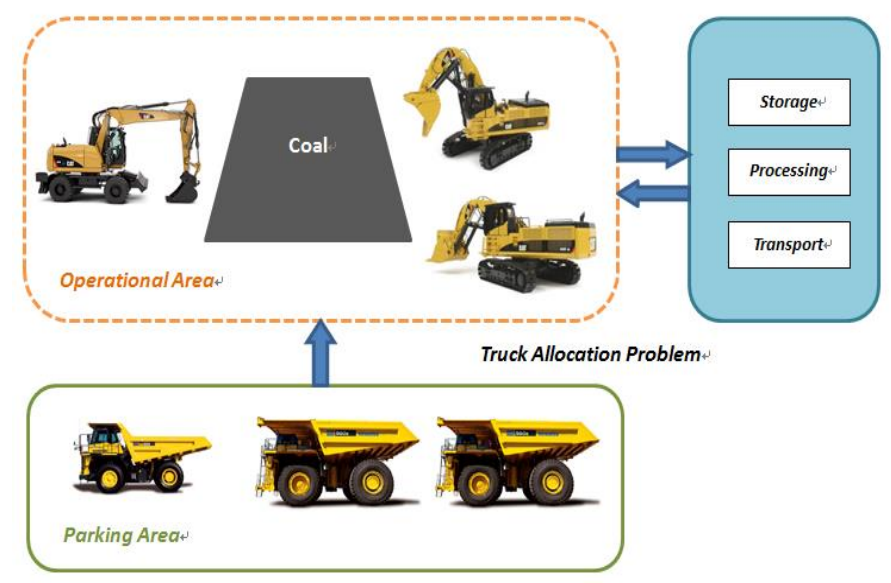

Fig. 1. Shovel-truck problem in coal mining.

Fig. 1 is the illustration of shovel production and haul truck allocation problem in coal mining operations. As shown in the figure, different types of trucks are dispatched from parking area to serve the shovels working in the operational area. Coal is extracted and loaded to haul trucks by shovels, and then they are transported to next operations for unloading, storage, processing and transport to customers or further use.
In this problem, two important decision variables needs to be determined. First is the shovel throughput, and the shovels should work at the level which can fulfill customer requirements on both quantity and quality. The other important decision is to determine which shovel is served by which haul truck or by which group of haul trucks. Several types of haul trucks with different loads are usually used in coal mining operations, so the truck allocation problem is a typical combinatorial optimization problem and can greatly affect the system performance. The purpose of this model is to determine optimal throughput of each shovel and truck allocation plan in order to improve the efficiency and utilization of shovels and trucks in coal mining operations while satisfy customer demands. Using this method, the operating cost and waste of in-process resources are minimized, further, the fuel consumption and GHG emission will also be reduced under the optimal operational plan.

\section{B. Model formulation}

The decision making model is formulated based upon mixed integer programming and aims to optimize the shovel throughput and truck allocation plan within a fixed period for coal mining operations. The main purpose of this model is to minimize the operating cost of shovel and haul truck operation.

Two sets and indices in the optimization model are first defined as, $T=1, \ldots, t$ is the set and index of haul trucks, and $S=1, \ldots, s$ is the set and index of shovels. The decision variables in this model are $T_{s}$ and $X_{s t} . T_{s}$ denotes the throughput of shovel $s$, and $X_{s t}$ is a binary decision variable determining whether truck $t$ is allocated to serve shovel $s$, and if $X_{s t}$ equals to 1 , truck $t$ is allocated to shovel $s$, and if $X_{s t}$ equals to 0 , otherwise. Besides, some parameters are also defined. $T L_{t}$ is defined as the load of haul truck $t$. $C G_{s}$ is defined as coal grade at shovel $s$. $U$ and $L$ represent the upper and lower limit of coal grade, respectively. $O_{t}$ is the operating cost of truck $t . D$ is defined as the customer demand of coal or the required daily production amount. $L O W_{s}$ and $U P P_{s}$ denote the lower and upper bound of shovel $s$, respectively. After all the necessary parameters and decision variables have been defined, the mathematical model for determining the optimal shovel throughput and truck allocation plan for coal mining operations is formulated.

\section{Minimize:}

$$
\text { Cost }=\sum_{t=1}^{T} O_{t} \cdot \sum_{s=1}^{S} X_{s t}+\sum_{s=1}^{S} O C_{s} T_{s}
$$

Eq. (1) is the objective function of the mathematical model, which minimizes the operational cost of coal mining activities including both shovel operating cost and haul truck operating cost. Seven constraints are also formulated in order to fulfill the capacity constraint, customer demand as well as other requirements.

\section{Subject to:}


The copy is the accepted manuscript by SIMS 2016.

The published version is available on IEEE Xplore: http://ieeexplore.ieee.org/document/7802905/

$$
\begin{gathered}
\sum_{s=1}^{S} T_{s} \geq D \\
\sum_{s=1}^{S} C G_{s} \cdot T_{s} \leq U \cdot \sum_{s=1}^{S} T_{s} \\
\sum_{s=1}^{S} C G_{s} \cdot T_{s} \geq L \cdot \sum_{s=1}^{S} T_{s} \\
L O W_{s} \leq T_{s} \leq U P P_{S}, \text { For } s=1, \cdots, S \\
\sum_{s=1}^{S} X_{s t} \leq 1, \text { For } t=1, \cdots, T \\
\sum_{t=1}^{T} T L_{t} \cdot X_{s t} \geq T_{s}, \text { For } s=1, \cdots, S \\
X_{s t} \in\{0,1\}, \text { For } s=1, \cdots, S ; t=1, \cdots, T
\end{gathered}
$$

Eq. (2) ensures the customer demand or the required amount of coal production should be fulfilled. Eqs. (3) and (4) guarantee the quality requirement of coal are satisfied. Constraint Eq. (5) is the capacity constraint that assures the throughput at shovel $s$ is less than its upper limit while more than its lower bound. The upper limit is the maximum capacity of shovel, and the lower bound requires a minimum output amount of shovel $s$ in order to avoid inefficient use of the shovel. Eqn. (6) guarantees haul truck $t$ can be allocated to at most to one shovel, and this avoid the allocation of one haul truck to multiple shovels in the same period. Eq. (7) guarantees that the all the throughput of the shovels can be served by haul trucks. Eq. (8) determines if shovel $s$ is served by haul truck $t$. Besides, all the variables are non-negative.

\section{ILLUSTRATION}

\section{A. Data assumption}

In this section, a numerical example is given for illustrating the application of the model for optimal planning of shovel production and truck allocation problem in coal mining. The example is assume to be a small-sized coal mining company in China where the quality of coal is categorized into six grades in accordance with sulfur content, as shown in Table I.

TABLE I. The GRAde of CoAl BASEd on Sulfur Content [13]

\begin{tabular}{|c|c|c|c|}
\hline Grade & Classification & Code & $\begin{array}{c}\text { Range of ash } \\
\text { content (\%) }\end{array}$ \\
\hline 1 & $\begin{array}{c}\text { Special low } \\
\text { sulfur coal }\end{array}$ & SLS & $\leq 0.50$ \\
\hline 2 & Low sulfur coal & LS & $0.51 \sim 1.00$ \\
\hline 3 & $\begin{array}{c}\text { Low medium } \\
\text { sulfur coal }\end{array}$ & LMS & $1.01 \sim 1.50$ \\
\hline 4 & $\begin{array}{c}\text { Medium sulfur } \\
\text { coal }\end{array}$ & MS & $1.51 \sim 2.00$ \\
\hline 5 & $\begin{array}{c}\text { Medium high } \\
\text { sulfur coal }\end{array}$ & MHS & $2.01 \sim 3.00$ \\
\hline 6 & High sulfur coal & HS & $>3.00$ \\
\hline
\end{tabular}

All the parameters in this example are assumed based on experiences. Three types of haul trucks with different truckload and operating cost are applied in the coal mining site, and the operating cost is directly proportional to the truckload. In the operational area, the haul trucks are allocated to serve four shovels, and all the relevant parameters related to shovels and haul trucks are given in Table II. In addition, we assume the grade of coal at shovels $1,2,3$ and 4 are 4, 3, 4 and 5, respectively. The upper limit of coal grade is 6 and the lower

\begin{tabular}{|c|c|c|c|c|c|c|c|c|c|c|c|c|}
\hline \multicolumn{13}{|c|}{ Parameters of haul trucks } \\
\hline$T$ & 1 & 2 & 3 & 4 & 5 & 6 & 7 & 8 & 9 & 10 & 11 & 12 \\
\hline$T L_{t}(\mathrm{t})$ & \multicolumn{4}{|c|}{3} & \multicolumn{4}{|c|}{4} & \multicolumn{4}{|c|}{5} \\
\hline$O_{t}(\mathrm{RMB})$ & \multicolumn{4}{|c|}{1000} & \multicolumn{4}{|c|}{1300} & \multicolumn{4}{|c|}{1500} \\
\hline \multicolumn{13}{|c|}{ Parameters of shovels } \\
\hline$S$ & \multicolumn{3}{|c|}{1} & \multicolumn{3}{|c|}{2} & \multicolumn{3}{|c|}{3} & \multicolumn{3}{|c|}{4} \\
\hline $\begin{array}{c}O C_{s} \\
(\mathrm{RMB} / \mathrm{t})\end{array}$ & \multicolumn{3}{|c|}{280} & \multicolumn{3}{|c|}{285} & \multicolumn{3}{|c|}{290} & \multicolumn{3}{|c|}{285} \\
\hline$C G_{s}$ & \multicolumn{3}{|c|}{4} & \multicolumn{3}{|c|}{3} & \multicolumn{3}{|c|}{4} & \multicolumn{3}{|c|}{5} \\
\hline$U P P_{s}$ & \multicolumn{12}{|c|}{5} \\
\hline$L O W_{s}$ & \multicolumn{12}{|c|}{18} \\
\hline \multicolumn{13}{|c|}{ Other parameters } \\
\hline$U$ & \multicolumn{12}{|c|}{6} \\
\hline$L$ & \multicolumn{12}{|c|}{3} \\
\hline$D(\mathrm{t} / \mathrm{d})$ & \multicolumn{12}{|c|}{42} \\
\hline
\end{tabular}
limit of coal grade is 3 . If the grade of coal is too high, the operational cost increases greatly, however, if the quality of coal is not good enough, the customer requirements cannot be fulfilled, so it is important to control the quality of coal at the right level.

TABLE II. PARAMETERS

* $\mathrm{RMB}$ is the unit of Chinese currency.

\section{B. Optimization and discussion}

In this example, we assume three managers are selected to determine the operational plan of coal mining activities. It is quite common especially in small- and medium-sized companies that the shovel production and haul truck allocation plan are determined based upon the experiences of the managers, and the experience-based decision-making may lead to non-optimal or sub-optimal solutions of the shovel-truck problem, which cause higher operational cost and more GHG emission.

TABLE III. OPERATIONAL PLAN A

\begin{tabular}{|c|c|c|c|c|}
\hline \multirow{2}{*}{ Shovel } & \multirow{2}{*}{$\begin{array}{c}\text { Throughput } \\
\boldsymbol{T} \boldsymbol{s}(\mathbf{t})\end{array}$} & \multicolumn{3}{|c|}{ Truck allocation } \\
\cline { 3 - 5 } & 13 & $\boldsymbol{T L}=\mathbf{3 t}$ & $\boldsymbol{T L}=\mathbf{4 t}$ & $\boldsymbol{T L}=\mathbf{5 t}$ \\
\hline$s=1$ & 10 & $t 4, t 3$ & & $t 9$ \\
\hline$s=2$ & 10 & & $t 5$ & $t 10$ \\
\hline$s=3$ & 9 & & $t 6, t 7$ & $t 11$ \\
\hline$s=4$ & & & $t 8$ & $t 12$ \\
\hline
\end{tabular}

TABLE IV. OPERATIONAL PLAN B

\begin{tabular}{|l|l|r|} 
Shovel & Truck allocation \\
\hline
\end{tabular}


The copy is the accepted manuscript by SIMS 2016.

The published version is available on IEEE Xplore: http://ieeexplore.ieee.org/document/7802905/

\begin{tabular}{|c|c|c|c|c|}
\hline & $\begin{array}{c}\text { Throughput } \\
\boldsymbol{T s}(\mathbf{t})\end{array}$ & $\boldsymbol{T L}=\mathbf{3 t}$ & $\boldsymbol{T L}=\mathbf{4 t}$ & $\boldsymbol{T L}=\mathbf{5 t}$ \\
\hline$s=1$ & 12 & $t 1, t 3$ & $t 6$ & $t 9$ \\
\hline$s=2$ & 13 & & $t 7$ & $t 11, t 12$ \\
\hline$s=3$ & 10 & $t 2, t 4$ & $t 8$ & \\
\hline$s=4$ & 10 & & $t 5$ & $t 10$ \\
\hline
\end{tabular}

\begin{tabular}{|c|c|c|c|}
\hline $\begin{array}{c}\text { The overall operating } \\
\text { costs (RMB) }\end{array}$ & 27155 & 29015 & 25140 \\
\hline Truck utilization & $87.8 \%$ & $88.2 \%$ & $100 \%$ \\
\hline
\end{tabular}

With the optimal operational planning, not only a better truck utilization rate (fewer haul trucks needed), but also a lower operating cost can be achieved. Table VI presents the comparison of the operational plans proposed by manager A, B and $\mathrm{C}$. Comparing with the first solution, the utilization rate of haul trucks improves by $100 \%-87.8 \%=12.5 \%$ and the overall operating cost reduces by $(27155-25140) / 27155=7.4 \%$ in the optimal operational plan, and comparing with the second one, the utilization of haul trucks improves by $100 \%-88.2 \%=11.8 \%$ and the overall operating cost reduces by (29105$25140) / 29105=13.6 \%$. The result has proved the effectiveness of the optimization model for shovel production and haul track allocation problem in surface coal mining industry. Through implementing the optimal operational plan, the shovel throughput can be optimized and the utilization rate of haul trucks can be improved, and furthermore, the environmental performance improves with the reduction of GHG emission. In addition, it is also noted the better performance is calculated upon daily or short term basis, and the benefits will be accumulated with time, which means much better economic and environmental benefits can be achieved in a long-term period.

\section{EXTENSION FOR ACCOUNTING UNCERTAINTIES OF EQUIPMENT FAILURE}

Literature shows the problem of equipment failure is one of the most significant challenges in mining operations [14-18]. Even if some advance methodologies, i.e., lean philosophy, proactive maintenance, etc., have been adopted in mining industry in order to reduce and minimize the rate of equipment breakdown, machine failure is still inevitable, and furthermore, the probability of equipment failure in mining industry is relatively higher than other industries due to the tough tasks they perform and the harsh environment where the equipment is operated.

Equipment failure is a stochastic event which is determined by several factors. However, the reliability and the rate of equipment failure of mining machines are time related and can be predicted using normal distribution formula and/or other mathematical methods [19]. Those methods provide theoretical foundation not only for prediction of mining equipment failure and proactive maintenance, but also for integrating the uncertainties associated with equipment failure in operational planning.

\section{A. Method}

Equipment failure is considered as uncertainty for mining operation, and the rate can be predicted. In this paper, we adopt the method developed by Birge and Louveaux [20] to incorporate the uncertainties of equipment failure with decision making in operational planning of surface coal mining.

First, let vector $p$ represents the integer variables and vector $q$ denotes the other variables. Let $V$ denotes the cost of haul truck allocation and vector $W$ includes all the other coefficients. Also, $\gamma=1 \ldots \emptyset$ are index and set of the possible scenarios. For scenario 
$\gamma$, the concise model is converted in Eq. (9). Herein, $m_{\gamma}$ is a vector and $M_{\gamma}, N_{\gamma}$ and $W$ are matrices.

$$
\begin{gathered}
\text { Minimize: } p V+q w_{\gamma} \\
M_{\gamma} q \leq m_{\gamma} \\
N_{\gamma} q \leq W p \\
p \in\{0,1\}, q \geq 0
\end{gathered}
$$

Assume the probability of scenario $\gamma$ is $f_{\gamma}$, and only a finite set of scenarios may happen within the studied period. The model can then be extended as shown in Eq. (10). It is noted that the optimal solution found in Eq. (10) is not the optimal solution for a single scenario, but it provide decisions for the worst-case scenario.

$$
\begin{gathered}
\text { Minimize: } p V+\sum_{\gamma=1}^{\emptyset} f_{\gamma} q_{\gamma} w_{\gamma} \\
M_{\gamma} q_{\gamma} \leq m_{\gamma} \\
N_{\gamma} q_{\gamma} \leq W p \\
p \in\{0,1\}, q \geq 0
\end{gathered}
$$

\section{B. Extended model and discussion}

Based upon the aforementioned method, the shovel production and haul truck allocation problem in surface coal mining operations is extended with consideration of the uncertainties related to shovel failure. In order to formulate the uncertain parameters, set $i=1 \ldots I$ is first defined as the possible scenarios of shovel failure. The relevant variables and parameters can then be redefined accordingly. $T_{s}^{i}$ is the shovel $s$ throughput of scenario $i, O C_{s}^{i}$ is the operational cost of shovel $s$ in scenario $i$. After the variables and parameters have been redefined, the extended model is presented in Eqs. (11)-(18).

\section{Minimize:}

$$
\text { Cost }=\sum_{t=1}^{T} O_{t} \cdot \sum_{s=1}^{S} X_{s t}+\sum_{i=1}^{I} \sum_{s=1}^{S} O C_{s}^{i} T_{s}^{i}
$$

\section{Subject to:}

$$
\begin{gathered}
\sum_{i=1}^{I} \sum_{s=1}^{S} T_{s}^{i} \geq D \\
\sum_{i=1}^{I} \sum_{s=1}^{S} C G_{s} \cdot T_{s}^{i} \leq U \cdot \sum_{i=1}^{I} \sum_{s=1}^{S} T_{s}^{i} \\
\sum_{i=1}^{I} \sum_{s=1}^{S} C G_{s} \cdot T_{s}^{i} \geq L \cdot \sum_{i=1}^{I} \sum_{S=1}^{S} T_{s}^{i}
\end{gathered}
$$

$$
\begin{gathered}
\operatorname{LOW}_{s} \leq \sum_{i=1}^{I} T_{s}^{i} \leq U P P_{s}, \text { For } s=1, \cdots, S \\
\sum_{s=1}^{S} X_{s t} \leq 1, \text { For } t=1, \cdots, T \\
\sum_{t=1}^{T} T L_{t} \cdot X_{s t} \geq \sum_{i=1}^{I} T_{s}^{i}, \text { For } s=1, \cdots, S \\
X_{s t} \in\{0,1\}, \text { For } s=1, \cdots, S ; t=1, \cdots, T
\end{gathered}
$$

The same numerical example in previous section is used to illustrate the impact of considering the uncertainties of equipment failure in surface coal mining operations. Three scenarios of equipment operation are considered in the example, which are normal operation, minor failure and serious failure. Under normal operation scenario, the production capacity reaches $100 \%$. Under minor failure scenario, small malfunctions will reduced the production capacity, but it is believed the malfunctions can be fixed within a short time so that there will not be a significant capacity loss in this scenario. Under serious failure scenario, the malfunctions need more time to be fixed, and this will lead to a significant capacity loss of production. The probability and percentage of production capacity with respect to different scenarios at each shovel is given in Table VII.

TABLE VII. THE PROBABILITY AND PRODUCTION CAPACITY OF THE SCENARIOS OF EQUIPMENT FAILURE

\begin{tabular}{|c|c|c|c|c|c|c|}
\hline \multirow{2}{*}{ Shovel } & \multicolumn{2}{|c|}{ Normal } & \multicolumn{2}{c|}{ Minor failure } & \multicolumn{2}{c|}{ Serious failure } \\
\cline { 2 - 7 } & Prob. & Yield & Prob. & Yield & Prob. & Yield \\
\hline$s=1$ & $75 \%$ & $100 \%$ & $20 \%$ & $80 \%$ & $5 \%$ & $10 \%$ \\
\hline$s=2$ & $75 \%$ & $100 \%$ & $15 \%$ & $80 \%$ & $10 \%$ & $10 \%$ \\
\hline$s=3$ & $70 \%$ & $100 \%$ & $25 \%$ & $85 \%$ & $5 \%$ & $15 \%$ \\
\hline$s=4$ & $80 \%$ & $100 \%$ & $15 \%$ & $80 \%$ & $5 \%$ & $10 \%$ \\
\hline
\end{tabular}

Based on the optimal operational plan in previous case, the overall coal production of this scenario reduces to 38 ton which cannot fulfill the production requirement or customer demand. Therefore, more amount of coal has to be produced and more resources are required in the production planning.

TABLE VIII. OPTIMAL PLAN

\begin{tabular}{|c|c|c|c|c|}
\hline \multirow{2}{*}{ Shovel } & Throughput & \multicolumn{3}{|c|}{ Truck allocation } \\
\cline { 3 - 5 } & $\boldsymbol{T s}(\mathbf{t})$ & $\boldsymbol{T L}=\mathbf{3 t}$ & $\boldsymbol{T L}=\mathbf{4 t}$ & $\boldsymbol{T L}=\mathbf{5 t}$ \\
\hline$s=1$ & 15 & $t 1, t 2, t 3, t 4$ & $t 6$ & \\
\hline$s=2$ & 8 & & $t 7, t 8$ & \\
\hline$s=3$ & 8 & & $t 5$ & $t 10$ \\
\hline$s=4$ & 15 & & & $t 9, t 11, t 12$ \\
\hline
\end{tabular}

The optimal planning for shovel production and truck allocation in this scenario is illustrated in Table VIII. The overall production becomes 46 tons in order to fulfill the customer demand. Besides, all the trucks are used and the truck utilization rate reduces to $95.8 \%$. 


\section{CONCLUDING REMARKS}

The paper has presented a novel approach for decision making of operational planning of surface coal mining. First, a typical shovel production and haul truck allocation problem is formulated, and the application of the model is illustrated through a numerical example. The model is then extended for taking into consideration of the probability of equipment failure which is one of the most important factors influencing the production capacity, and the model is re-formulated as a multiscenario mixed integer programming aiming at determining the optimal operational plan under the worst-case scenario. The result of the numerical calculation shows that more resources need to be used when the uncertainties of equipment failure is taken into account in order to guarantee the customer demand can be fulfilled.

The probability of equipment failure is time related and can be estimated based upon previous research results [20]. The most important contribution of this study is that the model considers the probability of equipment failure, and combines it with decision making of the operational planning of surface coal mining. This can provide decision makers with more realistic image of the challenges in operational planning of surface coal mining.

Limitations of this study and suggestions for future development are also discussed as follows:

1. The rate or probability of equipment failure is estimated based upon normal distribution theory or other mathematical distribution which are calculated in accordance with the statistics over a long period. However, the decision making in this model is for short period, and the probability has significant stochastic features, which may lead to more resource commitment to the optimal operational plan. Therefore, future development should be conducted addressing this problem.

2. In this paper, the operating cost is considered as the most important influencing factors in decision making. However, due to the preparation for the worst-case scenario when equipment failure is accounted, other types of cost such as maintenance cost, equipment repairing cost and inventory holding cost may be incurred, so further study should also consider those types of cost in decision making of optimal operational plan.

3. Literature has shown interests in different aspects other than economic efficiency for decision making of operational planning of surface coal mining. The future studies may consider expand the model to a multicriteria decision support system in order to account more objectives other than cost, i.e., risks [21, 22], environmental performance [22, 23], etc.

\section{ACKNOWLEDGMENT}

This research was carried out under the support from "Smart Mining Technology (SMT)" project (No. 2014-0083) financed by Utviklingsprogram of Narvik municipality.

\section{REFERENCES}

[1] C.H. Ta, J.V. Kresta, J.F. Forbes, and H.J. Marquez, "A stochastic optimization approach to mine truck allocation," International Journal of Surface Mining, Reclamation and Environment, vol. 19 (3), 2005, pp:162175.

[2] M.X. He, J.C, Wei, X.M, Liu, and B.X, Huang, "The genetic algorithm for truck dispatching problems in surface mine," Information Technology Journal, vol. 9 (4), 2010, pp: 710-714.

[3] T. Norgate, and N. Haque, "Energy and greenhouse gas impacts of mining and mineral processing operations," Journal of Cleaner Production, vol. 18, 2010, pp: 266-274.

[4] E. Siami-Irdemoosa, and S.R. Dindarloo, "Prediction of fuel consumption of mining dump trucks: A neural networks approach," Applied Energy, vol. 151, pp: 77-84.

[5] Y. Lizotte, and E. Bonates, "Truck and shovel dispatching rules assessment using simulation," Mining Science and Technology, vol. 5 (1), 1987, pp: 45-58.

[6] M.J.F. Souza, I.M. Coelho, S. Ribas, H.G. Santos, and L.H.C. Merchmann, "A hybrid heuristic algorithm for the open-pit-mining operational planning problem," European Journal of Operational Research, vol. 207, 2010, pp: 1041-1051.

[7] P.K. Mishra, M. Bolic, M.C.E. Yagoub, and R.F. Stewart, "RFID technology for tracking and tracing explosives and detonators in mining services applications," Journal of Applied Geophysics, vol. 76, 2016, pp: 33-43.

[8] L.K. Sahoo, S. Bandyopadhyay, and R. Banerjee, "Benchmarking energy consumption for dump trucks in mines," Applied Energy, vol. 113, pp: 1382-1396.

[9] E. Sun, A. Nieto, Z. Li, and V. Kecojevic, "An integrated information technology assisted driving system to improve mine trucks-related safety," Safety Science, vol. 48, 2010, pp: 1490-1497.

[10] M.J.F. Souza, I.M. Coelho, S. Ribas, H.G. Santos, and L.H.C. Merschmann, "A hybrid heuristic algorithm for the open-pit-mining operational planning problem," European Journal of Operational Research, vol. 207, 2010, pp: 1041-1051.

[11] M. Rodrigo, Z. Enrico, K. Fredy, A. Adolfo, "Availability-based simulation and optimization modeling framework for open-pit mine truck allocation under dynamic constraints," International Journal of Mining Science and Technology, vol. 2013, pp: 113-119.

[12] C.H. Ta, A. Ingolfsson, J. Doucette, "A linear model for surface mining haul truck allocation incorporating shovel idle probabilities," European Journal of Operational Research, vol. 223, pp: 770-778.

[13] Baidu library, 2015. The grade of coal related product. Available from: http://wenku.baidu.com/link?url=6Qz37-kQ_LkRe4e9tYkidrELx441SQ6-

lmt8EqLOiTgXCaZeIdjd_MV4In843i2MTKFerK9aPRuBkIDWey2sPb 3UdA3q4USZ1FskIedW_y (In Chinese, Retrieved on 10.12.2015)

[14] J. Barbady, and U. Kumar, "Reliability analysis of mining equipment: A case study of a crushing plant at Jajarm Bauxite Mine in Iran," Reliability Engineering \& System Safety, vol. 93, no. 4, 2008, pp: 647-653.

[15] N. Vagenas, N. Runciman, and S.R. Clement, "A methodology for maintenance analysis of mining equipment," International Journal of Surface Mining, vol. 11, no. 1, 1997, pp: 33-40.

[16] E. Dreyer, "Cost-effective prevention of equipment failure in the mining industry," International Journal of Pressure Vessels and Piping, vol. 61, no. 2-3, 1995, pp: 329-347.

[17] R.A. Hall, and L.K. Daneshmend, "Reliability modelling of surface mining equipment: Data gathering and analysis methodologies," International Journal of Surface Mining, Reclamation and Environment, vol. 17, no. 3, 2003, pp: 139-155.

[18] E. Topal, and S. Ramazan, "A new MIP model for mine equipment scheduling by minimizing maintenance cost," European Journal of Operational Research, vol. 207, 2010, 1065-1071.

[19] B.S. Dhillon, "Mining Equipment Reliability, Maintainability, and Safety," Springer, Berlin, 2008. 
The copy is the accepted manuscript by SIMS 2016.

The published version is available on IEEE Xplore: http://ieeexplore.ieee.org/document/7802905/

[20] J.R. Birge, and F. Louveaux, "Introduction of Stochastic Programming," Springer-Verlag, New York, 1997.

[21] H. Yu, W.D. Solvang, and S. Yuan, "A multi-objective decision support system for simulation and optimization of municipal solid waste management system," Proceeding of $3^{\text {rd }}$ International Conference on Cognitive Infocommunications, Kosice, pp: 193-199, 2012.
[22] H. Yu, W.D. Solvang, S. Yuan, and Y. Yang, "A decision aided system for sustainable waste management," Intelligent Decision Technologies, vol. 9 (1), 2015, pp: 29-40.

[23] H. Yu, W.D. Solvang, and C. Chen, "A green supply chain network design model for enhancing competitiveness and sustainability of companies in high north arctic regions," International Journal of Energy and Environment, vol. 5 (4), 2014, pp: 403-418. 